\title{
V1432 Aql: A polar or an intermediate polar?
}

\author{
K. P. Singh ${ }^{1}$ and V. R. Rana ${ }^{1,2}$ \\ 1 Department of Astronomy \& Astrophysics, Tata Institute of Fundamental Research, Homi Bhabha Road, Colaba, \\ Mumbai 400005, India \\ e-mail: singh@tifr.res.in, vrana@tifr.res.in \\ 2 Joint Astronomy Programme, Department of Physics, Indian Institute of Science, Bangalore 560012, India
}

Received 19 February 2003 / Accepted 25 July 2003

\begin{abstract}
We present evidence for a spin period of $4070 \mathrm{~s}$ in a magnetic cataclysmic variable V1432 Aql, classified as a polar. The evidence is based on the analysis of archival X-ray data obtained from a $25 \mathrm{ks}$ long and uninterrupted observations with $X M M-$ Newton. We also detect modulation of the X-ray flux with the orbital period, various harmonics of the spin period, and side-bands due to beating of the spin period with the orbital period. The energy-dependent light curves and hardness ratios show a very complex behaviour with both the spin phase and the orbital phase, indicating the presence of multiple absorbers of different densities. The observed characteristics suggest the presence of a disk in the system and accretion via a disk-overflow mechanism as is commonly observed in intermediate polars.
\end{abstract}

Key words. accretion, accretion disks - stars: binaries: close - stars: novae, cataclysmic variables stars: individual: V1432 Aql - X-rays: stars

\section{Introduction}

V1432 Aql was discovered to be a Magnetic Cataclysmic Variable (MCV) during X-ray imaging observations of the Seyfert Galaxy NGC 6814 with ROSAT by Madejski et al. (1993). Before its discovery V1432 Aql was confused with the nearby Seyfert Galaxy NGC 6814 which is $37^{\prime}$ away, and a period of $12100 \mathrm{~s}$ seen in X-rays was attributed (erronously) to NGC 6814 (Mittaz \& Branduardi-Raymont 1989; Done et al. 1992; Leighly et al. 1994). The brighter of the two X-ray sources in the ROSAT field of view, V1432 Aql has been subsequently classified as an eclipsing polar binary system. Based on extensive optical observations and analysis of X-ray data it is believed to contain a magnetic white dwarf accreting from the Roche-Lobe of a late-type companion via an accretion channel or an accretion curtain with perhaps multiple foot-prints on the white dwarf (Staubert et al. 1994; Watson et al. 1995; Patterson et al. 1995; Geckeler \& Staubert 1997; Friedrich et al. 2000). The eclipses are probably caused either by the accretion stream or the secondary star (Schmidt \& Stockman 2001; Geckeler \& Staubert 1999). Being an eclipsing system, its binary period is well established. The presence of sharp dips in the optical and X-ray light curves, and radial velocities reveal that the orbital period of the system is $12116.3 \mathrm{~s}$ (Patterson et al. 1995). The spin period associated with the white dwarf in V1432 Aql has been somewhat controversial, however. From the changing nature of the accretion region relative to the

Send offprint requests to: V. R. Rana, e-mail: vrana@tifr.res.in magnetic pole, Geckeler \& Staubert (1997) estimate the white dwarf spin period as $12150 \mathrm{~s}$ based on X-ray (ROSAT) and optical photometry. V1432 Aql is, therefore, generally believed to be an asynchronous polar with the unusual characteristic of having a rotation (or spin) period of the white dwarf that is longer than its orbital period around the companion star. On the other hand, Mukai (1998) has presented evidence for the presence of a spin period of $\sim 4040 \mathrm{~s}$ based on archival ROSAT $\mathrm{X}$-ray data. Additional characteristics that further distinguish V1432 Aql from the normal AM Her systems are the complexity of its X-ray light-curves and very strong hard X-ray component (Singh et al. 2003), which are more common in the intermediate polars (IPs).

Currently, only four nearly synchronous polars are known (Mouchet et al. 1999). These are V1500 Cyg, BY Cam, V1432 Aql and RXJ2115-5840. The spin period of the white dwarf is usually less than its orbital period, the exception being V1432 Aql. Therefore V1432 Aql has always been considered as unique in this group of "nearly synchronous polars". Furthermore, the difference between the white dwarf spin period and the orbital period $(<0.3 \%)$ is the smallest in the group, resulting in a beat period of about 50 days. Geckeler \& Staubert (1997) have argued that the white dwarf spin period decreases by $\sim 9.8 \times 10^{-9} \mathrm{~s} \mathrm{~s}^{-1}$ which implies that the system will synchronize in $\sim 100$ yrs.

Several doubts about the above picture of V1432 Aql have been raised by Mukai (1998) who pointed out various problems with the asynchronous model for V1432 Aql. Based on 
X-ray observations, Mukai also presented an alternate model in which V1432 Aql is an intermediate polar with a spin period near 4040s. However, all previous X-ray observations (with the exception of EXOSAT) have been with the near-Earth satellites with interruptions casued by the Earth's shadowing. The maximum continuous exposure in such observations is typically an hour or less, making it difficult to see periods around this value clearly. V1432 Aql was recently observed with XMMNewton which can observe sources uninterruptedly for a long time. Here, we present our analysis of archival X-ray data on V1432 Aql obtained with XMM-Newton. The paper is organised as follows: In the next section (Sect. 2) we present observational details, followed by analysis and results in Sect. 3, where we also present continuous X-ray light curves in four energy bands and their discrete Fourier transforms, and folded $\mathrm{X}$-ray intensity and the hardness ratio profiles as a function of phase. A discussion is presented in Sect. 4 followed by conclusions in Sect. 5.

\section{Observations}

V1432 Aql was observed with XMM-Newton (Jansen et al. 2001) on 9 October 2001. XMM-Newton has 3 large X-ray telescopes with the largest effective area of any X-ray satellite, and an optical monitor (OM, Mason et al. 2001) for simultaneous optical and X-ray observations. The observations were carried out with the EPIC cameras containing the MOS CCDs (Turner et al. 2001) with a thin filter used to block contamination by visible and UV light. An exposure time of $25455 \mathrm{~s}$ was realized with each of the two cameras - MOS1 and MOS2 operating in Timing mode, also known as Fast mode. In this mode, applied only to the central CCD, 100 rows in a central window with a width of 100 pixels are compressed into one dimension which is parallel to the CCD readout register to give fast time slices of the incoming X-ray events (see Turner et al. 2001). A timing resolution of $1.75 \mathrm{~ms}$ per time slice is available in the Fast mode. Note that Fast mode is different from the "small window" mode where a window of $100 \times 100$ is read out with a time resolution of $0.3 \mathrm{~s}$. MOS 1 and MOS2 are orthogonal, so two projections at right angles were available. There is no information on the background rates in the central CCD in the Fast mode. The high resolution spectroscopy detectors with an energy range of $0.3-2.0 \mathrm{keV}$, RGS1 and RGS2 (den Herder et al. 2001), were operated in standard spectroscopy mode. Data from OM were not available.

\section{Analysis and results}

We extracted good photon events (FLAG $=0$ ) from the central CCD of both MOS1 and MOS2 with patterns 0-12 (see Fig. 7 of Turner et al. 2001a), CCD columns between 280 and 340 , and energies between $0.2-12 \mathrm{keV}$, and with $1 \mathrm{~s}$ time bins to produce X-ray light curves in the "total" energy band of $0.2-$ $12 \mathrm{keV}$. The CCDs contained a noisy first few columns and a repeated pattern every 8 th column which contribute most of the counts below $200 \mathrm{eV}$. Therefore, the lowest energy threshold was kept at $200 \mathrm{eV}$, and thus all such noise was completely removed from the data. Similarly, X-ray light curves were also extracted in 3 smaller energy bands, viz., $0.2-0.5 \mathrm{keV}$ ("soft"), 0.5-2.0 keV ("medium") and 2-12 keV ("hard"), to study the energy dependence of X-ray emission. The energy bands were chosen such that the soft and medium bands correspond to the ROSAT soft and hard bands respectively. This selection of bands also gives sufficient counts to define hardness ratios (see Sect. 3.2), and provides a crude separation of various spectral components that are relevant here. All the X-ray light curves from MOS1 and MOS2 were examined individually and found to show nearly identical behaviour except for their mean count rate. The light curves corresponding to the same energy band from MOS1 and MOS2 were averaged to further improve the statistics for timing analysis. Since MOS1 and MOS2 are nearly identical, the averaging was done by simply adding the binned counts in the MOS1 and MOS2 and then dividing by two. The light curves, thus obtained in four energy bands - total, soft, medium and hard, were rebinned into $50 \mathrm{~s}$ time bins and are shown in 4 panels in Fig. 1. Strong and nearly periodic variability is clearly seen in all the energy bands, with the modulation increasing with energy. Further analysis of these light curves is presented below.

Total energy spectra were also accumulated for both MOS1 and MOS2 using the good events as explained above. X-ray spectra are seen to be very hard and show well resolved line emission at $6.4,6.7$ and $7.0 \mathrm{keV}$ presumably from the flourescence of $\mathrm{Fe}$ and plasma emission from highly ionised $\mathrm{Fe}$. A preliminary analysis of the average spectrum shows that it is consistent with an unabsorbed soft component, an absorbed multi-temperature plasma component with a very low Fe abundance, and a hard reflection component. A detailed spectral analysis of the data will be presented elsewhere. The counts in RGS were insufficient for a meaningful spectral analysis.

\subsection{X-ray light curves and power spectra}

Discrete Fourier transforms were performed on the extracted $\mathrm{X}$-ray light curves with $1 \mathrm{~s}$ time resolution. The data were detrended by subtracting the mean count rates prior to performing the Fourier transformations. Thus power spectra (sums of the mean-square-amplitudes of the detrended data) were obtained for data in the four energy bands for MOS1, MOS2 individually, and the averaged data from both MOS1 and MOS2, and found to show identical characteristics in the corresponding energy bands. Consequently, power spectra only for the averaged light curves in 4 energy bands in Fig. 1 are shown in Fig. 2. The highest peak seen in all the power spectra corresponds to a period of $\sim 4070 \mathrm{~s}$ in all the energy bands. This frequency is shown marked as $\omega$ in Fig. 2. The error in the determination of the fundamental period is $\sim 160 \mathrm{~s}$. Significant power is also observed at the frequency corresponding to the well known orbital period of $12116.304 \mathrm{~s}$, shown as $\Omega$ in Fig. 2. Both the frequency components, $\omega$ and $\Omega$, dominate at all the energies. It should be noted, however, that the present data are not able to distinguish between the orbital period of $12116.304 \mathrm{~s}$ and the previously known spin period of $12150 \mathrm{~s}$. However, since the orbital period of $12116.304 \mathrm{~s}$ is very well established and correctly predicts the eclipses in the present data (see Sects. 3.3,4), 


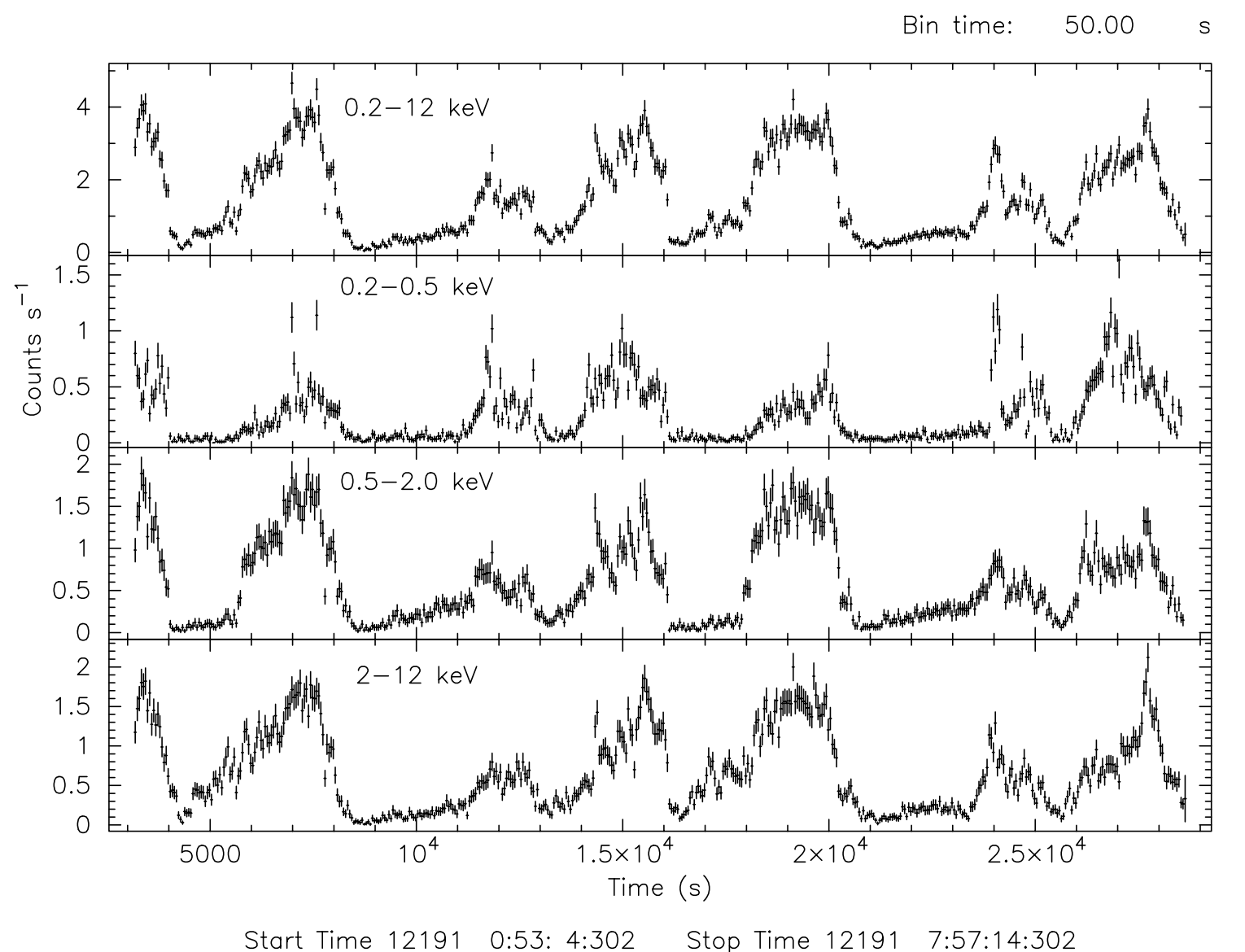

Fig. 1. X-ray light-curves of V1432 Aql in 4 different energy bands as indicated in the panels, and obtained by combining (averaged) data from EPIC MOS1 and MOS2 cameras aboard the XMM-Newton satellite. The bin time is $50 \mathrm{~s}$ in all the four panels. The observations were carried out on 9 October 2001. The start and stop times are in units of TJD.

we attribute the peak seen near this period to the orbital period of the system. The dominant peak near the period of $4070 \mathrm{~s}$ is then most likely due to the spin period of the white dwarf in the system.

The noise level in the power spectra has been determined using a procedure described by Norton et al. (1996). The absolute amplitude of the power level above which at least $N / 10$ number of noise peaks are present, where $N$ is the number of independent frequency samples, was estimated. The value of $N$ can vary according to the length of observation and the time resolution of the light curve from which power spectrum has been obtained. This estimated value of noise level corresponds to a $90 \%$ confidence limit, since there is only a $10 \%$ chance that the peak above this level is due to noise. The noise level in the power spectra was thus found to be nearly constant in the frequency band shown and at a level of $\sim 0.002$ (counts $/ \mathrm{s})^{2}$ in the total energy band and $\sim 0.0015$ (counts $/ \mathrm{s})^{2}$ in the other three energy bands. Several peaks, in addition to the two peaks discussed above, can be seen above the noise in the power spectra. Some of these peaks have been identified either as harmonics of $\omega$ or due to beating between the two frequencies $\omega$ and $\Omega$. These are $2 \omega, 3 \omega, \omega-\Omega, \omega+\Omega, \omega-2 \Omega, \omega+2 \Omega, 2 \omega-\Omega, 2 \omega+\Omega$, $3 \omega-\Omega$, and $3 \omega+\Omega$. Some of these components happen to be too close to each other to be distinguishable. For example, both $\Omega$ and $\omega-2 \Omega$ are confused, and so are $\omega+2 \Omega$ and $2 \omega-\Omega$.
Similarly, the frequency components $2 \Omega$ and $\omega-\Omega$ are very close, and so are $3 \Omega$ and $\omega$. Most of the frequency components are best seen in the total energy band. The peak positions of various frequency components in the narrower energy bands are, however, consistent considering the error in the determination of $\omega$. Frequency components corresponding to $\omega-\Omega$ and $\omega+\Omega$ are seen to be the 3 rd most dominant in nearly all the energy bands.

We also subtracted a sinusoidal component corresponding to $\omega$ from the light curve and then carried out the power spectral analysis. The peak corresponding to the $\omega$ completely disappeared whereas the remaining peaks gained a little more power. The major peak, identified as due to the spin $\omega$ is, therefore, unlikely to be due to $3 \Omega$.

\subsection{Spin folded $X$-ray light curves}

We have used the most dominant period derived by us to fold the X-ray data and see its behaviour as a function of phase of this period. The folded light curves in the soft, medium and hard bands defined above are shown in 3 panels of Fig. 3. The starting epoch is arbitrary and taken to be $\operatorname{HJD}\left(T_{\mathrm{s}}\right)=$ 2452 191.5. These profiles show a bright and a faint structure that are easy to interpret as arising from the spin of the white dwarf with a dominant accreting pole, as is commonly seen in 


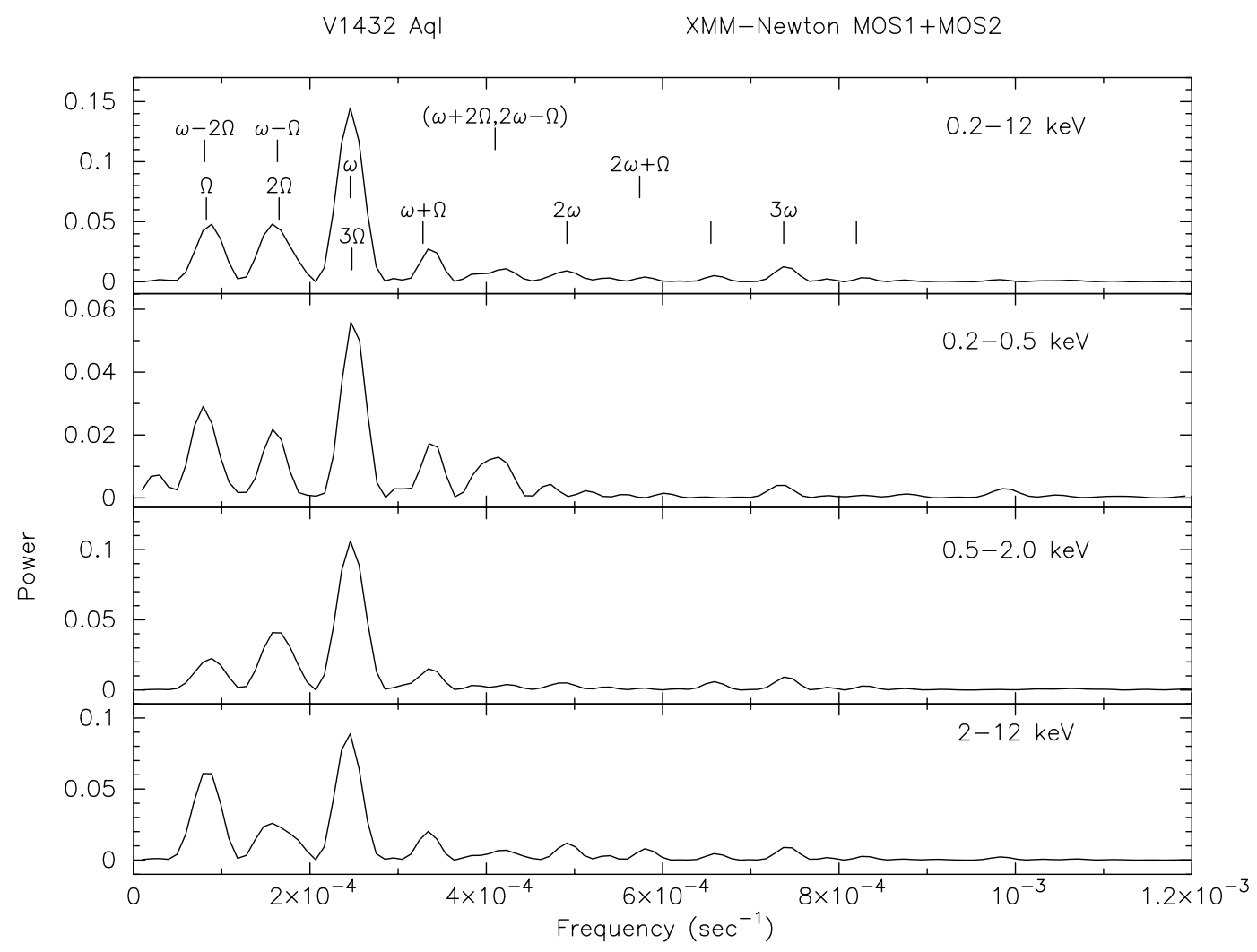

Fig. 2. Discrete power spectrum of V1432 Aql corresponding to data shown in Fig. 1. Energy bands shown are total, soft, medium, and hard, arranged in panels from top to bottom. The dominant frequency component corresponding to the spin of the system is shown marked as $\omega$, and the frequency component corresponding to the orbital period is shown as $\Omega$. The harmonics and side-band frequencies expected from the beating of $\omega$ and $\Omega$, and detected above the noise level are also indicated with tick-marks and labels.

many polars. Therefore, this period of $4070 \mathrm{~s}$ may well correspond to the rotation of the white dwarf in the system. An asymmeteric bright phase ( $\phi \simeq 0.4-1.0)$ is seen in all the energy bands. Similar profiles were reported by Mukai (1998) after folding the ROSAT data on the spin period of $4040 \mathrm{~s}$ derived by him. Three distinct peaks at $\phi \simeq 0.53,0.66-0.74$ and 0.82 can be seen during the bright phase in the "medium" and "hard" energy bands. These peaks in the "medium" and "hard" energy bands coincide with dips in the "soft" energy band. These, and other similar effects, give rise to an interesting pattern in the hardness ratio variations with respect to the intensity variations (see below).

Hardness ratio curves obtained from the 3 energy bands are shown plotted as a function of the spin phase in 2 panels in Fig. 4. Two hardness ratios have been defined - HR1 and HR2, where HR1 is the ratio of the count rate in $0.5-2.0 \mathrm{keV}$ to the count rate in $0.2-0.5 \mathrm{keV}$ energy bands, and HR2 is the ratio of the count rate in $2-12 \mathrm{keV}$ to the count rate in $0.5-2.0 \mathrm{keV}$ energy bands. The HR1 ratio shows small dips and peaks for $\phi=0.0-0.23$, and several big peaks beyond that phase range. HR1 appears to be the highest for $\phi=0.23-0.45$ when the intensity is almost minimum in all the energy bands. The hardness ratio $\mathrm{HR} 2$, on the other hand, shows a broad asymmetric maximum centered at $\phi \simeq 0.30$ and covering the phase range of $0.0-0.4$. This behaviour shows that the faint phase is most likely due to a strong absorption effect with maximum absorption between $\phi=0.23-0.45$. Between $\phi=0.4-1.0$, HR2 is very low and nearly constant. Several peaks seen in HR1 during this phase range conicide with dips in the soft band intensity, with the most prominent feature of this kind being at $\phi=0.8$. Such features could either indicate the presence of several low density absorbing clouds in the line of sight to the brighter accreting pole, or a complex magnetic field structure of the bright pole.

\subsection{Orbital modulation}

We have used the orbital period of $12116.304 \mathrm{~s}$ to fold the data and study its behaviour as a function of the binary orbit. The starting epoch is based on the ephemeris given by Watson et al. (1995) and is: $\operatorname{HJD}\left(T_{\mathrm{o}}\right)=2449217.9235(5)+0.1402350(1) E$. The folded light curves in the soft, medium and hard bands defined above are shown in 3 panels of Fig. 5. Hardness ratios as defined above are shown plotted as a function of the orbital phase in 2 panels in Fig. 6.

Basically three broad maxima and minima are seen in Fig. 5. One of the minima is near an eclipse at phase 0.98 and is seen clearly in all the energy bands and is even better defined in the HR1 and HR2 profiles (Fig. 6). The eclipse appears to be equally deep in all the energy bands, and is nearly total. Another minima/dip is near phase 0.35 , which has also been seen in the hard X-ray observations with the RXTE in 2002 but not in 1998 (Rana et al. 2002; Singh et al. 2003). The third minima/dip is near $\phi=0.6$. This dip is very sharp in the hard 


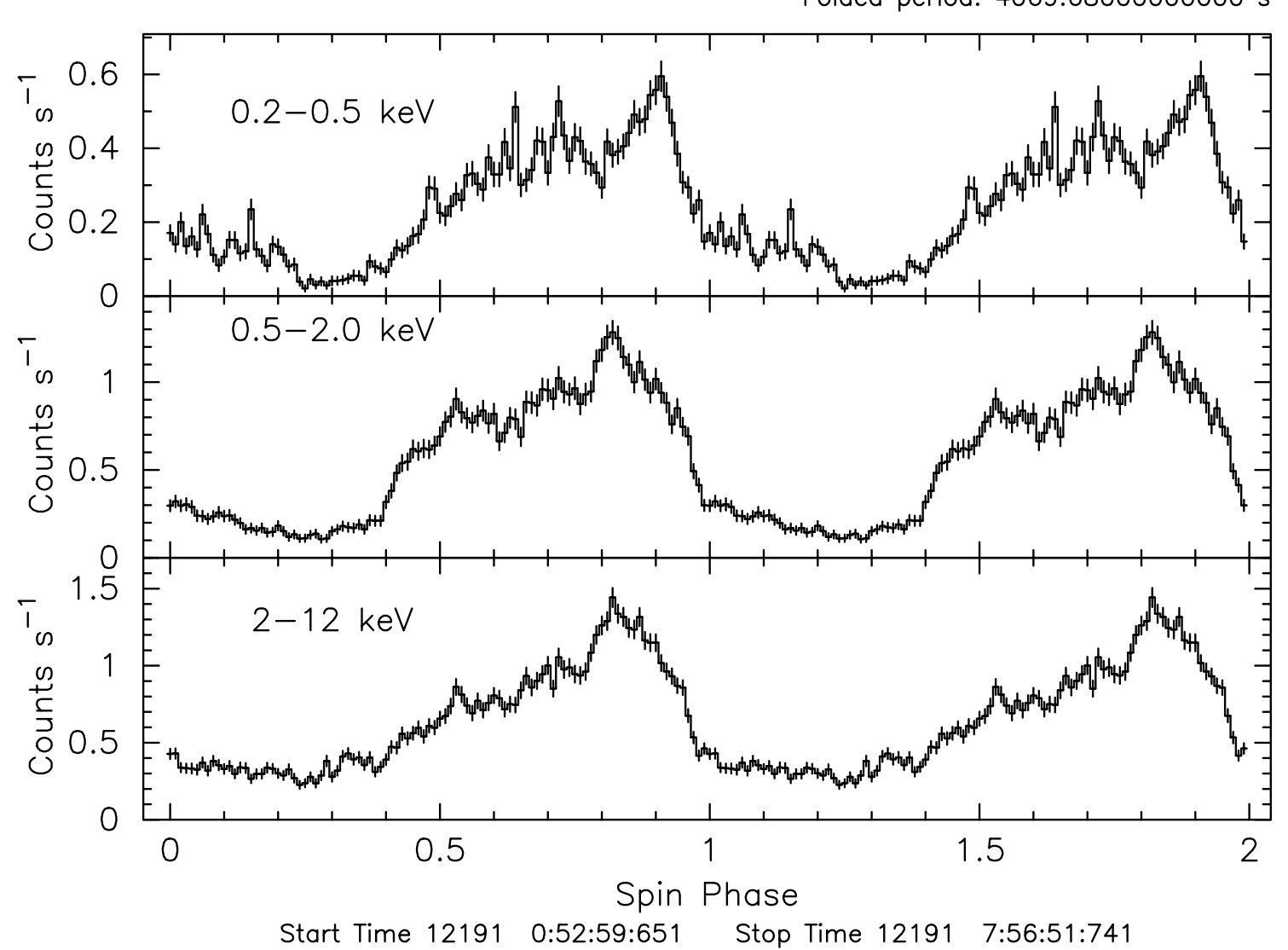

Fig. 3. X-ray light-curves folded on the spin period of $4069.68 \mathrm{~s}$ derived from data in Fig. 1 are shown for 3 energy bands of "soft" (0.2$0.5 \mathrm{keV})$, "medium" $(0.5-2 \mathrm{keV})$ and "hard" (2-12 keV) X-rays, arranged from top to bottom respectively. The spin period is repeated for a better appearance.

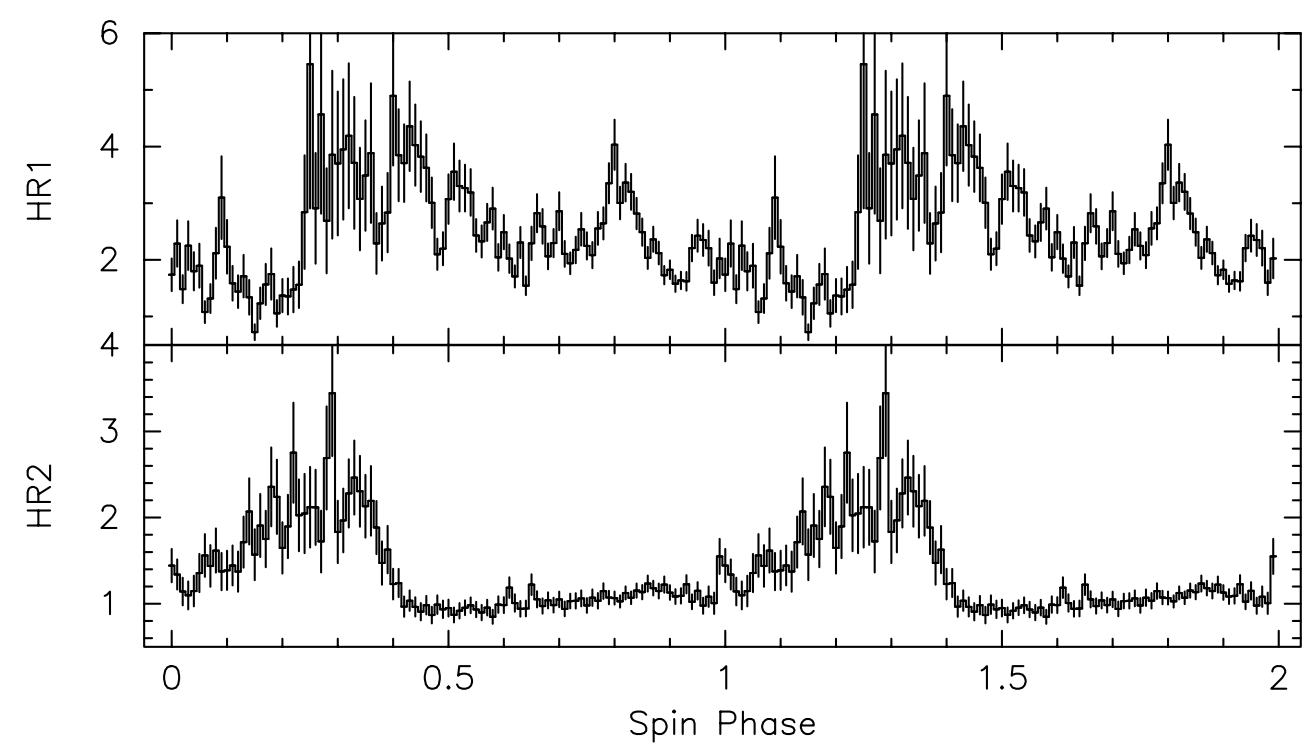

Fig. 4. X-ray hardness ratios, HR1 and HR2 (top to bottom) as defined in the text, are shown folded on the spin period. The spin period profile is repeated as before.

energy band, but broader and smoother in the softer energy bands. It appears as if a bright region that is heavily covered at phase 0.6 rapidly becomes transparent to hard X-rays above $2 \mathrm{keV}$, though it remains opaque to the lower energy X-rays until $\phi=0.7$ where the spectrum is the hardest (see HR2 in Fig. 6). After this phase the soft X-rays are also observed and the hardness ratio, HR2, settles down to a relatively lower value and coincides with the maximum in the soft X-ray emission. 
Folded period: $12116.3040000000 \mathrm{~s}$

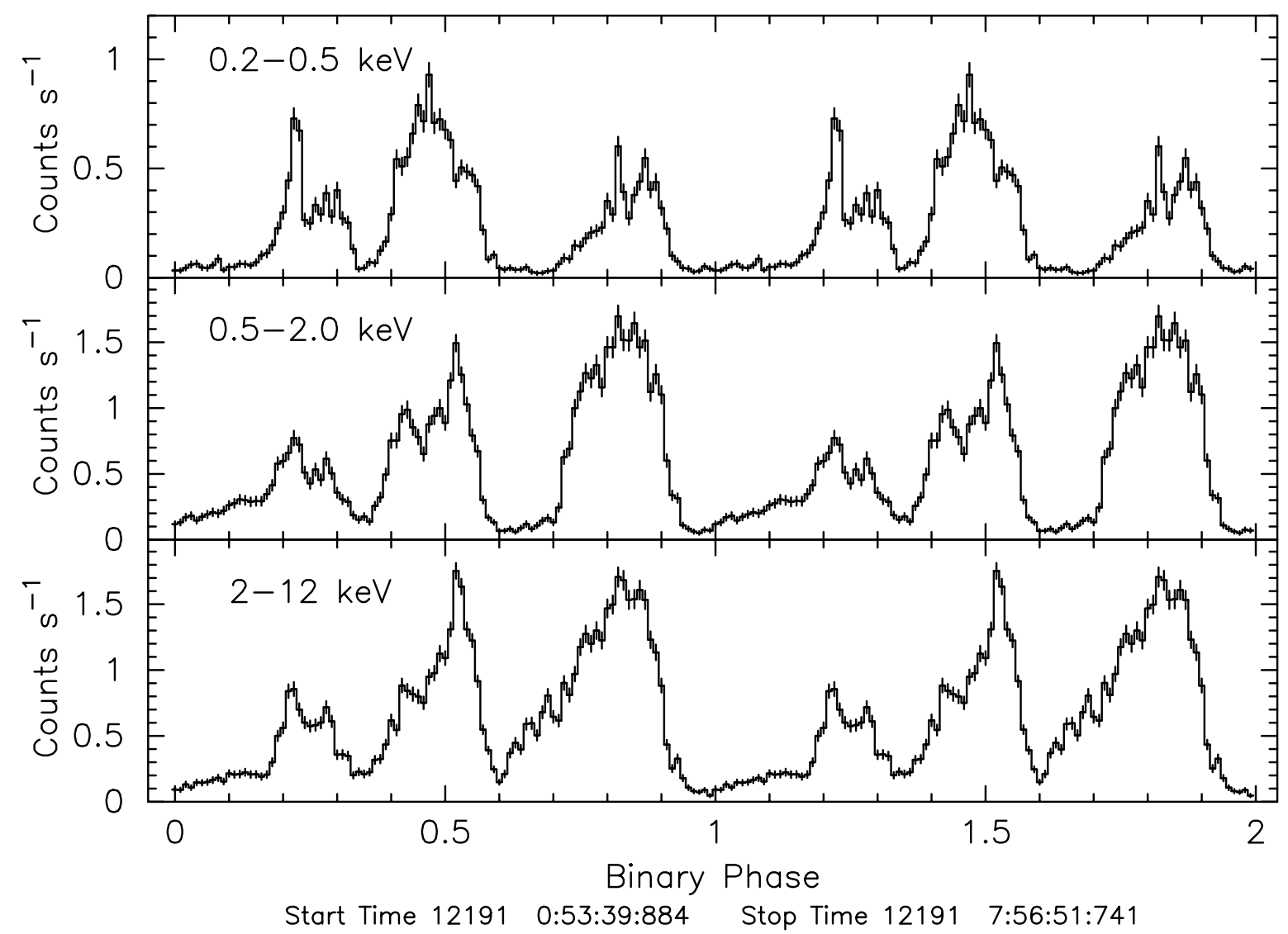

Fig. 5. X-ray light-curves folded on the well known orbital period of 12116.304 s for V1432 Aql are shown for "soft", "medium" and "hard" energy bands, from top to bottom respectively. The orbital period is shown twice for a better appearance.

The dip at $\phi=0.6$ was only hinted at in the RXTE observations, because of the lack of complete coverage with the RXTE.

Each of the three broad maxima in Fig. 5 show either a double or a triple peak structure at energies below $2 \mathrm{keV}$, which appear to merge into a single asymmetric peak in the hard band. Of the 3 peaks, the leading peak (just after the rising portion of the intensity) in each case is the strongest in the soft band, whereas the trailing peak (just before the falling portion of the intensity) is the stongest in the harder energy bands. Consequently the structure in the HR1 hardness ratio produces a complicated pattern with several peaks, whereas HR2 has a simpler structure with only three broad maxima. The maxima in HR2 (binary phase $0.58-0.72$, and 0.92-0.98) do not coincide with the peaks in HR1, rather they correspond to minima in HR1, and in total intensity. This behaviour can be understood if clouds of different densities are present along the line of sight, with the densest and perhaps the coldest clouds giving rise to maxima in HR2 and the lower density clouds leading to peaks in HR1.

As already pointed out, the hardness ratio, HR2, is very low during the third intensity maximum at binary phase between $0.72-0.92$ (Fig. 6). This is most probably indicative of the spectral hardness of the X-ray continuum.

\section{Discussion}

$X M M$-Newton has provided the longest continuous and most sensitive observation of V1432 Aql covering nearly 2 orbital cycles of the binary star system. A frequency component, $\omega$, corresponding to a period of $4070 \mathrm{~s}$ is found to dominate the power spectra in all energy bands. The second most common feature in the power spectra is the presence of a broad component centered very close to the orbital frequency of the system. Although these observations are not able to resolve various other frequency components that seem to be present in the power spectra, several peaks can be explained as harmonics of the most dominant frequency component and its side-bands arising from beating with the well known orbital period. The spin folded light curves are easy to explain if $\omega$ is associated with the spin of the white dwarf. The spin period can also be seen directly in the X-ray light curves in Fig. 1. The spin amplitude, however, changes significantly from one spin cycle to another in Fig. 1. It is quite common to see such a behaviour in the IPs, for example, in TX Col (Schlegel \& Salinas 2003), and TV Col (Rana et al. 2003).

The X-ray light curves when folded over the orbital period of the system indicate correctly the eclipse phase to be near 0.98 as is seen in all previous optical and X-ray observations with RXTE in 1998 and 2002 (Singh et al. 2003). This indicates that the power seen at $\Omega$ can be ascribed to the orbital 


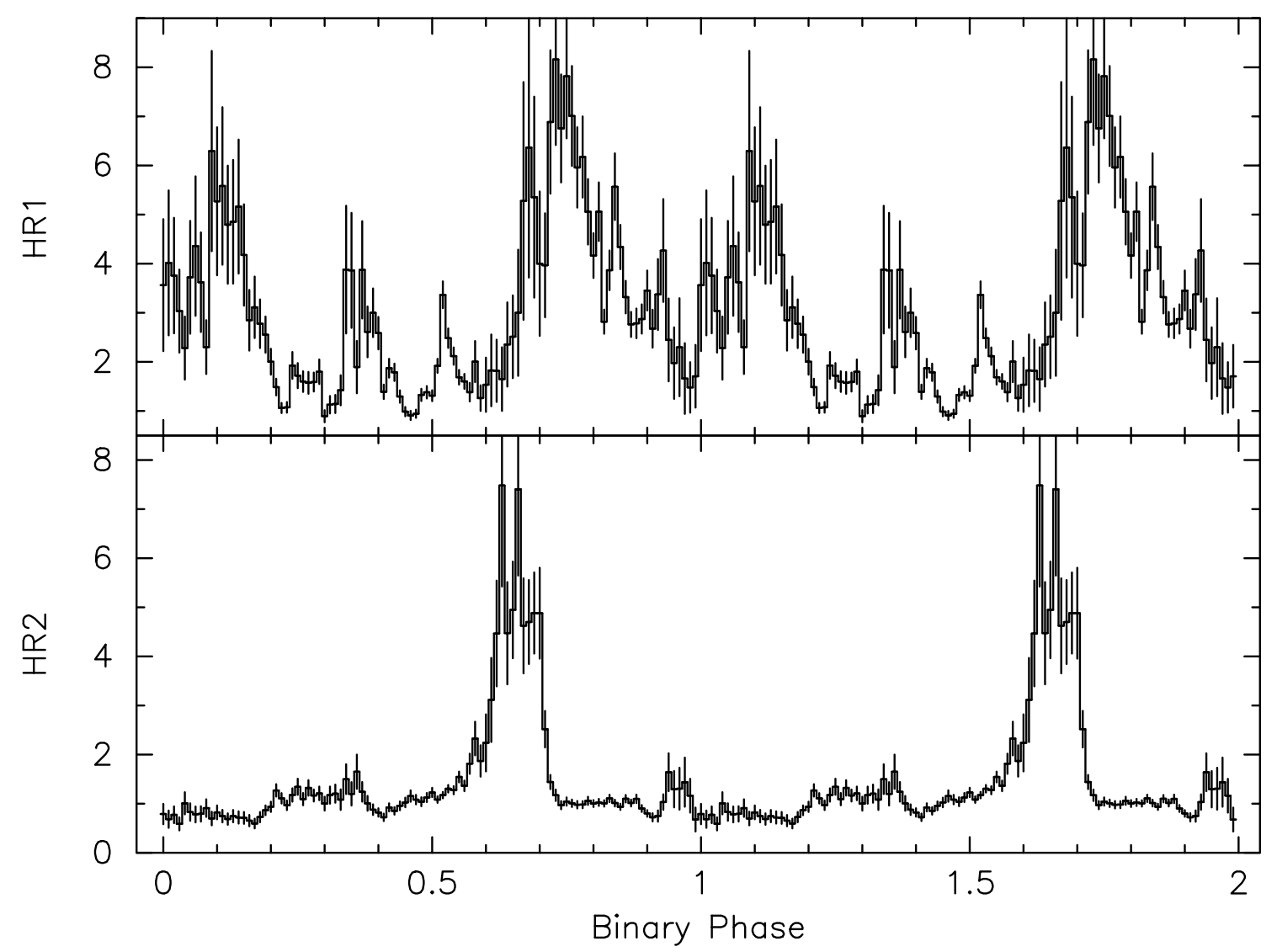

Fig. 6. X-ray hardness ratios, HR1 and HR2 (top to bottom) as defined in the text, are shown folded on the orbital period. The orbital period profile is repeated as before.

period of $12116.3 \mathrm{~s}$ rather than the period at $12150 \mathrm{~s}$ which was previously believed to represent the spin of the system.

The observed combination of the frequency components is expected naturally in a system that is accreting from the diskoverflow geometry where both an accretion disk and an accretion stream may be present (see Norton et al. 1996). In particular, the dominance of $\omega$ in the power spectra and the presence of its harmonics at all energies (see Fig. 2) suggest the existence of a disk in the system. The presence of both $\omega-\Omega$ and $\omega+\Omega$ with nearly the same power also indicates the presence of a disk-fed system as seen in the IPs. The hard X-ray spectra seen here and with the RXTE (Singh et al. 2003) are also more commonly seen in the IPs. The complex behaviour of the spectral hardness ratios, both with the spin phase and the orbital phase, points to multiple absorbers in the system. Several dips accompanied by increased hardness ratio could be due to the interaction of the accretion stream with the disk resulting in the material being splashed out of plane and forming cool clouds that absorb X-rays produced close to the white dwarf (see Frank et al. 1987).

Extensive optical photometry of V1432 Aql carried out so far and reported in the literature has not revealed any periodicity at the spin period reported here. It is, however, not unusual that the spin period of the white dwarf in IPs discovered in $\mathrm{X}$-ray observations is not clearly seen in the optical photometry. For example, TV Col, which is among the first IPs to be discovered in X-rays with a spin period of $1910 \mathrm{~s}$, has failed to reveal such a period in the optical over the last 20 years (see Retter et al. 2003).

\section{Conclusions}

1. The most dominant frequency component in the power spectra of X-ray light curves of V1432 Aql, in several energy bands between $0.2-12 \mathrm{keV}$, corresponds to a period of $4070 \mathrm{~s}$, and is most likely due to the spin of the white dwarf.

2. The second most important feature in the power spectra of V1432 Aql is most probably due to the orbital period of $12116.3 \mathrm{~s}$ that predicts the X-ray eclipse correctly in the data folded on this period.

3. The presence of several harmonics of the spin frequency, and its side-bands due to interference with the orbital frequency suggests accretion due to disk-overflow in the system.

4. The present observations suggest that V1432 Aql may be an intermediate polar rather than a polar.

5. Multiple absorbers with different densities are indicated to be present in the system.

Acknowledgements. We thank Matteo Guainazzi for helping us with the SAS tools for the analysis of data from XMM-Newton. This work is based on observations obtained with $X M M-N e w t o n$, an ESA science mission with instruments and contributions directly funded by ESA member states and NASA in USA. We thank Franco Giovannelli for his useful comments on the paper. 


\section{References}

den Herder, J. W., Brinckman, A. C., Kahn, S. M., et al. 2001, A\&A, 365, L7

Done, C., Madejski, G. M., Mushotzky, R. F., et al. 1992, ApJ, 400, 138

Frank, J., King, A. R., \& Lasota, J.-P. 1987, A\&A, 178, 137

Friedrich, S., Schwarz, R., Schwope, A., et al. 2000, in Abstracts of the Annual Scientific Meeting of the Astronomische Gesellschaft, Bremen, Sep. 18-23, 2000, vol. 17, p. 66

Geckeler, R. D., \& Staubert, R. 1997, A\&A, 325, 1070

Geckeler, R. D., \& Staubert, R., 1999, in Abstracts of the Annual Scientific Meeting of the Astronomische Gesellschaft, Goettingen, 20-25 Sep. 1999, vol. 15, p. 47

Jansen, F., Lumb, D., Altieri, B., et al. 2001, A\&A, 365, L1

Leighly, K., Kunieda, H., Tsusaka, Y., Awaki, H., \& Tsuruta, S. 1994, ApJ, 421, 69

Madejski, G. M., Done, C., Turner, T. J., et al. 1993, Nature, 365, 626

Mason, K. O., Breeveld, A., Much, R., et al. 2001, A\&A, 365, L36

Mittaz, J. P. D., \& Branduardi-Raymont, G. 1989, MNRAS, 238, 1029

Mouchet, M., Bonnet-Bidaud, J.-M., \& de Martino, D. 1999, ASP Conf. Ser., On Magnetic Cataclysmic Variables (AnnapolisMaryland), ed. C. Hellier, \& K. Mukai, 157, 215
Mukai, K., 1998, ApJ, 498, 394

Norton, A. J., \& Watson, M. G. 1989, MNRAS, 237, 853

Norton, A. J., Beardmore, A. P., \& Taylor, P. 1996, MNRAS, 280, 937

Patterson, J., Skillman, D. R., Thorstensen, J., \& Hellier, C. 1995, PASP, 107, 307

Rana, V. R., Singh, K. P., \& Barrett, P. 2002, In Proceedings of Multi Colour Universe Conf. at TIFR, Mumbai, 11-14 September 2001, ed. R. K. Manchanda \& B. Paul, 67

Rana, V. R., Singh, K. P., Schlegel, E. M., \& Barrett, P. 2003, ApJ, accepted

Retter, A., Hellier, C., Augusteijn, T., et al. 2003, MNRAS, 340, 679

Schlegel, E. M., \& Salinas, A. 2003, in ASP Conf. Ser., on Magnetic Cataclysmic Variables, IAU Colloq. 190 (Capetown) ed. M. Cropper \& S. Vrielmann

Schmidt, G. D., \& Stockman, H. S. 2001, ApJ, 548, 410

Singh, K. P., Rana, V. R., Mukerjee, K., Barrett, P., \& Schlegel, E. M., 2003, in ASP Conf. Ser. on Magnetic Cataclysmic Variables, IAU Colloq. 190 (Capetown) ed. M. Cropper \& S. Vrielmann

Staubert, R., Koenig, M., Friedrich, S., et al. 1994, A\&A, 288, 513

Turner, M. J. L., Abbey, A., Arnaud, M., et al. 2001, A\&A, 365, L27

Watson, M. G., Rosen, S. R., O’Donoghue, D., et al. 1995, MNRAS, 273, 681 